\title{
ARTHUR B. McDONALD - A RECENT NOBEL LAUREATE
}

\author{
ROBY AUSTIN* \\ Department of Astronomy and Physics \\ Saint Mary's University, 923 Robie Street, Halifax, NS B3K 3C3
}

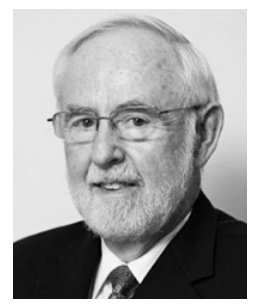

Dr. Arthur B. McDonald

Arthur B. McDonald was born in 1943 in Sydney, Cape Breton Island. His parents, Bruce and Valerie, were from families of Scottish and French settlers. Art's father, who was a lieutenant in the Canadian Army, went to Europe not long after Art was born to participate in the liberation of Holland, where he was both wounded and decorated for bravery. Art's youth was spent in Sydney, where he attended the Sydney Academy and was an active member of the YMCA youth service club, the Hi-Y. It was through the Hi-Y that he met Janet, his wife of (so far) 50 years. At 17, Art left Cape Breton (but not Nova Scotia) to study physics at Dalhousie University in Halifax. He was an enthusiastic student and obtained both a B.Sc. and an M.Sc. from Dalhousie. For his Ph.D., Art decided to travel farther afield and in 1965, he travelled west to the California Institute of Technology, from which he graduated with his doctoral degree in 1969. After his doctoral work, he was employed at AECL's Chalk River laboratories in Northern Ontario for several years, after which he was appointed as faculty at Princeton University. It was while he was at Princeton

\footnotetext{
* Author to whom correspondence should be addressed: Roby Austin E-mail: austin@ap.smu.ca

1 "Arthur B. McDonald - Biographical". Nobelprize.org. Nobel Media AB 2014. Web. 20 Dec 2016. <nobelprize.org/nobel_prizes/physics/laureates/2015/mcdonald-bio.html.
} 
that he began work on the project that eventually became the Sudbury Neutrino Observatory (SNO). In 1989 he moved from Princeton to Queen's University in Kingston Ontario, where he remained for the rest of his career and is emeritus faculty.

Neutrinos are subatomic particles which interact only weakly with other matter. They are not electrically charged and they do not carry any of the quark's form of charge, colour. When neutrinos were first discovered, they were thought to be massless. No experiment provided any evidence that neutrinos had mass, and at the beginning there were no theories that included neutrino mass. There are known to be three different kinds ("flavours") of neutrinos: electron neutrinos, muon neutrinos, and tau neutrinos. One significant source of the neutrinos that we can observe on earth is our sun. They are a product of some of the nuclear reactions that are the sun's bread and butter. The neutrinos produced in the sun's reactions are one flavour: electron neutrinos.

The first experiments to measure the flux of neutrinos from the sun detected many fewer neutrinos than were expected. The sun's luminosity indicates how many nuclear reactions must be taking place there, and for some reason, the neutrinos produced in those reactions were not being observed here on earth. This became known as "the solar neutrino problem", which, ultimately, Art McDonald and his collaborators at (SNO) lab, with his co-Nobellists at the Super-Kamiokande lab in Japan, were able to resolve.

Because neutrinos only interact very weakly with matter, it takes a lot of looking to detect neutrinos. Neutrino detectors are generally very large volumes. The neutrino detector at SNO lab, $2 \mathrm{~km}$ below the surface of the earth in an INCO nickel mine, was an engineering feat. In an active, full-time operating mine, the laboratory was maintained at standards of cleanliness that are the envy of many above-ground laboratories. The detector itself consisted of an acrylic vessel several stories high, filled with heavy water and surrounded by 9500 tremendously sensitive light sensors. When there was a rare interaction between a neutrino and some of the water in the tank, the upshot would be a little flash of light in the deep underground darkness. The light sensors recorded the pattern of the flash, and from that the scientists in the SNO collaboration were able to deduce the presence of neutrinos from the sun. 
The great brilliance of the SNO detector was to borrow 1000 tonnes of heavy water from Atomic Energy of Canada Limited (AECL). This one apparently simple adaptation allowed the SNO detector to be sensitive to the other kinds of neutrinos as well as electron neutrinos. Heavy water is chemically the same as water, but the hydrogen atoms have an extra nucleon; each hydrogen atom is twice its normal weight. Heavy water is rare (and expensive) but since CANDU reactors use it, AECL had a stockpile that the SNO project could use for a while. Filling their acrylic vessel with heavy water permitted the scientists at SNO to distinguish the light flash pattern that could only be caused by electron neutrinos and that which was caused by any of the three flavours.

Years of hard and clever work on the part of the SNO collaboration, with Dr. McDonald in the lead, permitted two measurements of the neutrino flux from the sun: One measurement was the electron neutrino flux only and the other was the sum of all (including the electron neutrinos). If neutrinos were massless, then the electron neutrinos created in the sun would be electron neutrinos when they reached the earth and no other flavours of neutrinos would be detected - the electron-neutrino-only flux would be equal to the all-flavours-of-neutrinos flux. However, the two were not equal. There were other flavours of neutrinos arriving at the SNO laboratory on earth that had originated in nuclear reactions in the sun.

The sun's nuclear reactions only produced electron neutrinos, so they were changing flavour during the journey between the sun and the earth. Such a remarkable thing, changing flavour, requires neutrinos to have mass. A massless neutrino simply cannot change flavour. So the work by the scientists at SNO, under Dr. McDonald's direction, revealed that the resolution to the solar neutrino problem was that neutrinos are massive particles.

It remains unknown what the mass of the neutrino is. It is known to be very small, and there are some details known about the relationships between the masses of the different flavours of neutrinos. The measurement of the mass of the neutrino will be another enormous scientific undertaking involving many collaborators and complicated and clever equipment. And perhaps the leader of that effort will also be a very pleasant Caper, a bright spark from Sydney making an invaluable contribution to science, so important that it merits recognition from the Nobel committee. 
